\title{
Effects of Verapamil and Its Optical Isomers on Repetitive Slow Responses Induced by Electrical Depolarization in Canine Ventricular Myocardium
}

\author{
Tetsunori Saikawa, M.D.* and Makoto Arita, M.D.
}

\section{SUMMARY}

The electrophysiological effects of verapamil (raccmic compounds) and its optical ( $\mathrm{d}$ - and $\mathrm{l}-$ ) isomers on canine ventricular myocardial fibers were investigated in current clamp conditions using single sucrose gap chamber and microelectrodes. The current-voltage (I-V) relationships were obtained in normal and low $\mathrm{Na}(12 \mathrm{mM})$-low $\mathrm{Ca}(0.45 \mathrm{mM})$ solutions with and without the drugs. Verapamil and its optical isomers blocked repetitive action potential discharges (slow responses) induced by depolarizing DC-currents. However, l-isomer was more potent than $\mathrm{d}$-isomer in suppressing these responses. The difference in the potency was attributed to their different actions on the steady state I-V relationships. Namely, l-isomer increased time independent membrane conductance to potassium ions (probably $\mathrm{g}_{\mathrm{k} 1}$ ), while d-isomer did not. This effect of l-isomer may favor the suppression of phase 4 depolarization and hence reduce the frequency of repetitive action potential discharges in depolarized ventricular muscle more effectively than d-isomer.

\section{Additional Indexing Words:}

Ca-antagonist Verapamil Optical isomer Automaticity Outward current Papillary muscle Depolarization Single sucrose gap Slow response

ERAPAMIL has been known as a "specific" slow channel inhibitor since Kohlhardt et al"1) reported that the agent suppressed "specifically" the slow inward current in cat papillary muscle. In Purkinje fibers, however, it has become evident that so-called "calcium antagonists" like $\mathrm{Mn}^{++}$and D 600 had multiple rather than selective effects on the ionic channels. These agents abolished the slow inward currents and modified the outward $\mathrm{K}+$ currents in the plateau phase. ${ }^{8)}$ Japan.

From the Department of Physiology, Faculty of Medicine, Medical College of Oita, Oita 879.56,

Address for reprint: Dr. Makoto Arita, Department of Physiology, Faculty of Medicine, Medical College of Oita, Oita 879-56, Japan.

* Present address: First Department of Medicine, Faculty of Medicine, Kyushu University, Fukuoka 812

Received for publication July 30, 1979. 
The effects of $d$ - and l-optical isomers of verapamil have also been studied in cardiac muscle preparations by several investigators, ${ }^{3), 4), 10,, 13}$ but the results were somewhat conflicting, especially concerning the effects of the d-isomer. For example, Bayer et al ${ }^{3,4}$ ) claimed that d-isomer had little effect on the slow inward current, whereas Kaumann et $\mathrm{al}^{10)}$ and Raschack ${ }^{13)}$ reported that $\mathrm{d}$-isomer had essentially the same effects as l-isomer in suppressing contractile tension as well as in inhibiting experimental arrhythmias.

These somewhat puzzling results prompted us to study the effects of $d$ and l-isomers of verapamil on the repetitive (automatic) slow responses produced in electrically depolarized ventricular myocardium, because the development of such repetitive responses in ventricular muscle has been attributed to activation of the slow inward currents. ${ }^{1), 2,, 7), 9), 14}$ )

In the present study we first investigated the effect of racemic verapamil on the repetitive slow responses produced by electrical depolarization in canine ventricular papillary muscle. We then compared the effect of the l-isomer on repetitive responses with that of the d-isomer, since there was no rcport describing such effects of these isomers. Both d-and l-isomer suppressed the repetitive responses, but the potency of the 1-isomer was greater than that of the d-isomer. The potency difference was at least in part attributed to different actions of these isomers on the steady state current-voltage relationship.

\section{Methods}

Ventricular papillary muscles $(0.8-1.0 \mathrm{~mm}$ in diameter; $5-7 \mathrm{~mm}$ in length) were excised from right and left ventricles of adult mongrel dogs anesthetized with intraperitoneal injections of sodium pentobarbital $(40 \mathrm{mg} / \mathrm{Kg})$. The details of the single sucrose gap method used in the present study are essentially the same as those which appeared in the previous paper. ${ }^{2}$ In brief, the preparations were mounted in a 3-compartment single sucrose gap chamber (volume $0.6 \mathrm{ml}$ ). Conventional microelectrodes filled with $3 \mathrm{M} \mathrm{KCl}$ were used to record transmembrane potentials from the preparation. Driving stimuli and/or depolarizing DC-currents for induction of repetitive slow responses were applied through the sucrose gap $(1.8 \mathrm{~mm}$ in width). The intensity of applied DC-currents of 4-5 sec duration, membrane potentials and the first derivative of the membrane potential $\left(\dot{V}_{\max }\right)$ were displayed simultaneously on an oscilloscope (Nihon Khoden VC-9A) and ink-writing recticorder (Nihon Kohden). For control records, preparations were perfused with Tyrode solution $\left(\mathrm{NaCl} 137, \mathrm{KCl} 2.7, \mathrm{CaCl}_{2} 1.8, \mathrm{MgCl}_{2} 1.0, \mathrm{NaHCO}_{3} 12.0, \mathrm{NaHPO}_{4}\right.$ 0.4 , and Glucose $5.0 \mathrm{mM} / \mathrm{L}$ ) at a flow rate of $1.5 \mathrm{ml} / \mathrm{min}$. Verapamil (racemic compounds) and its optical isomers (d- and 1 -) were diluted to concentrations of 1 and $6 \mu \mathrm{g} / \mathrm{ml}$ with Tyrode solution and these test solutions were introduced into the test chamber to study the effects. In some experiments $\mathrm{NaCl}$ was totally substituted for isomolar choline chloride and the concentration of $\mathrm{CaCl}_{2}$ was reduced to $0.45 \mathrm{mM}$ (low $\mathrm{Na}-\mathrm{Ca}$ solution). All solutions were warmed $\left(36^{\circ} \mathrm{C} \pm 0.5^{\circ} \mathrm{C}\right.$ ), 
aerated with a mixture of $95 \% \mathrm{O}_{2}$ and $5 \% \mathrm{CO}_{2}$, and maintained at $\mathrm{pH} 7.3$. Uniform current clamp was achieved by limiting the length of the preparation within the test chamber to less than $1 \mathrm{~mm}$. The uniformity of the potential distribution during current clamp was checked by recording membrane potentials at both proximal and distal portions of the preparation simultaneously, and the uniformity was satisfactory.

\section{RESULTS}

The effects of racemic verapamil on the electrically induced repetitive action potential discharges (slow responses) were tested. Since the effects of low concentrations $(1 \mu \mathrm{g} / \mathrm{ml})$ of the agents were not remarkable, high concentrations $(6 \mu \mathrm{g} / \mathrm{ml})$ were used in the following experiments. Fig. 1 A-C shows control repetitive slow responses induced by depolarizing DC-currents of $5 \mathrm{sec}$ duration at 3 different membrane potential levels. The ionic mechanism of such slow automatic responses in canine ventricular muscle has been studied elsewhere. ${ }^{1,2)}$ After a $20 \mathrm{~min}$ application of verapamil the action potential discharge rate was markedly decreased in association with decreased $\dot{\mathrm{V}}_{\max }$ and overshoot (D, E, F). After 40 min of application, the action potential discharges completely disappeared as shown in the right panels $(G, H, I)$. In Fig. $2 A$ and $B$, the cycle length, $\dot{V}_{\max }$ and overshoot of the responses ob-

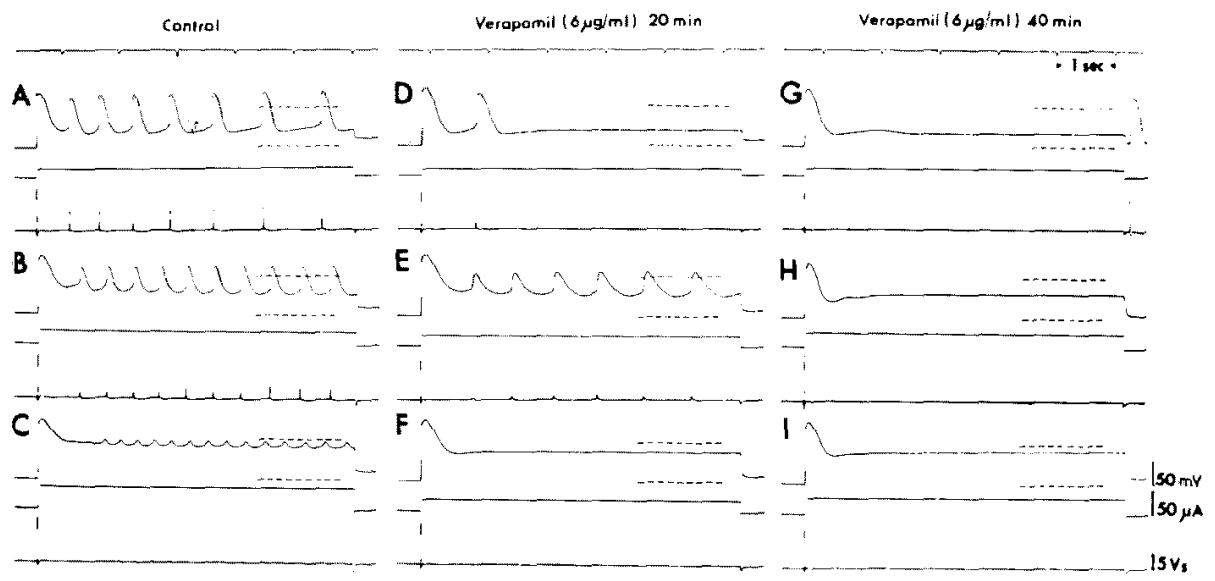

Fig. 1. Effect of verapamil (racemic compounds) on repetitive action potential discharges induced by depolarizing DC-currents in canine ventricular papillary muscle. Left panels $(A, B, C)$ show control records at 3 different membrane potentials. Middle panels $(D, E, F)$ are $20 \mathrm{~min}$ after application of the drug $(6 \mu \mathrm{g} / \mathrm{ml})$; Right panels $(\mathrm{G}, \mathrm{H}, \mathrm{I}), 40 \mathrm{~min}$ after application. Top traces: 1 sec tirne marks. Panels top trace: membrane potentials; middle trace: applied current; bottom trace: first derivative of membrane potential $\left(\dot{V}_{\max }\right)$. Broken lines in each panel denote $0 \mathrm{mV}$ and resting potential. 

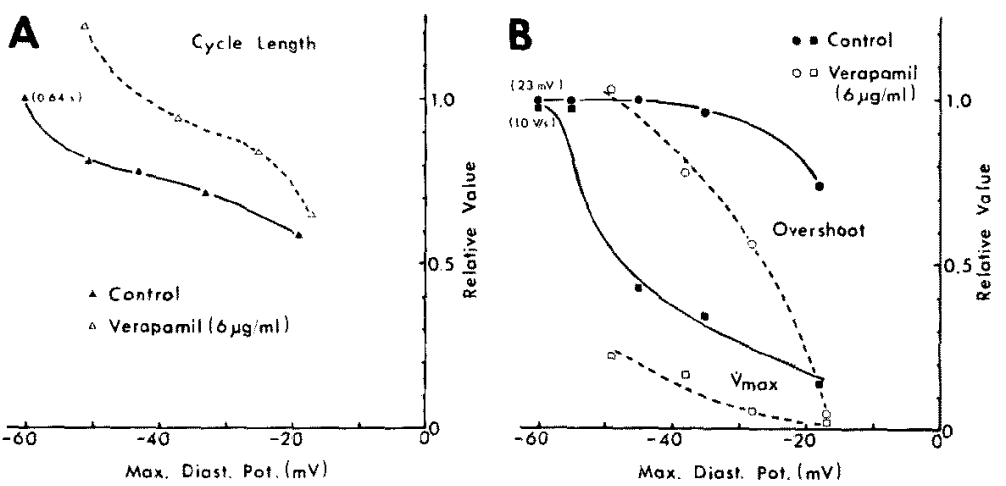

Fig. 2. Effect of verapamil (racemic compounds) on relation between maximum diastolic potentials and cycle length (A), and $\dot{V}_{\max }$ and overshoot of the repetitive action potentials (B). In both $A$ and $B$ abscissae indicate membrane potentials; ordinates, change relative to control value (unity) measured at membrane potential of $-60 \mathrm{mV}$ before drug application. Solid symbols and solid lines denote control records; open symbols and broken lines, records $10-12 \mathrm{~min}$ after application of drug $(6 \mu \mathrm{g} / \mathrm{ml})$. Each curve fitted by eye.

tained after 10-12 min of the drug application are plotted in comparison with those before drug application. During drug action, the cycle length of the action potential discharges was prolonged $(\mathrm{A})$, and $\dot{\mathrm{V}}_{\max }$ and overshoot were decreased markedly (B). The decrease of overshoot and $\dot{V}_{\max }$ appeared much larger in a range of less negative membrane potentials.

The mechanism of the inhibitory action of verapamil on repetitive slow responses of this kind has been attributed to a reduction of slow inward currents. ${ }^{2,9)}$ However, an alternative possibility could be modification of the background outward currents, e.g., $i_{k 1 .}{ }^{12}$ ) In order to test this possibility we investigated the effect of verapamil on the steady state $\mathrm{I}-\mathrm{V}$ relation in preprations perfused with low $\mathrm{Na}-\mathrm{Ca}$ solution. Perfusion with this solution strongly inhibited repetitive action potential discharges induced by depolarizing currents, and hence facilitated measurement of steady state $\mathrm{I}-\mathrm{V}$ relations before and after application of the drugs.

Fig. 3 shows results of one such experiment in a low $\mathrm{Na}-\mathrm{Ca}$ medium. Panels $\mathrm{A}$ and $\mathrm{B}$ represent control records without verapamil at 2 different intensities of applied current. Panels $\mathrm{C}$ and D were obtained during perfusion with verapamil, and show that the magnitude of depolarization attained by the same intensity of depolarieing current is much smaller than seen in panels $\mathrm{A}$ and $\mathrm{B}$. This suggests an increase in membrane conductance.

Fig. 4 shows the relationships between applied current and resulting steady membrane potential (I-V relation) which were obtained from experiment such as those shown in Fig. 3, but in another preparation. The mem- 


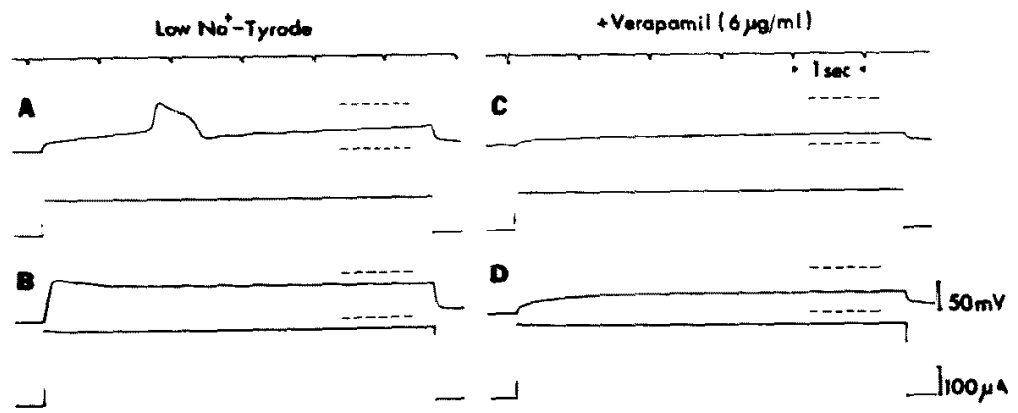

Fig. 3. Effect of verapamil (racemic compounds) on membrane potential in low $\mathrm{Na}(12 \mathrm{mM})$-low $\mathrm{Ca}(0.45 \mathrm{mM})$ media. $\mathrm{A}$ and $\mathrm{B}$ recorded before, $\mathrm{C}$ and $\mathrm{D}$ recorded after application of drug $(6 \mu \mathrm{g} / \mathrm{ml})$. Top traces : $1 \mathrm{sec}$ time marks. Panel top traces: membrane potential; bottom traces: applied current. Broken lines in each panel denote $0 \mathrm{mV}$ and resting potential.

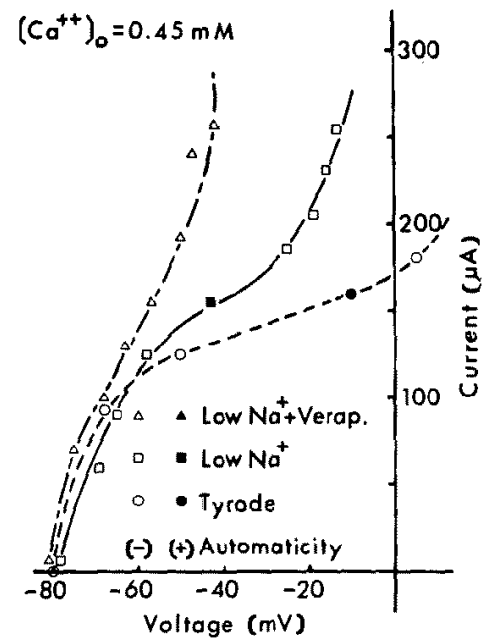

Fig. 4. Steady state current-voltage relation (open symbols) obtained in current clamp condition during perfusion with normal Tyrode solution (circle), low $\mathrm{Na}^{+}(12 \mathrm{mM})-$ low $\mathrm{Ca}^{++}(0.45 \mathrm{mM})$ solution without verapamil (square), and Tyrode solution plus racemic verapamil (triangle). Solid symbols mean at least one automatic action potential was elicited during passage of depolarizing current of $5 \mathrm{sec}$ duration, in which the average of maximum diastolic potentials was tentatively used as the "steady" membrane potential in abscissa. Each curve fitted by eye.

brane potentials were measured at the end of the depolarizing current pulses. It can be seen that anomalous (inward going) rectification is present in the voltage range from -60 to $0 \mathrm{mV}$ in normal Tyrode solution. Similar $\mathrm{I}-\mathrm{V}$ relations have been demonstrated in cardiac Purkinje fibers immersed in Na-deficient solutions with the use of current clamp methods. ${ }^{6}$ ) The curve 
of $\mathrm{I}-\mathrm{V}$ relation shifted upward and to the left after changing the perfusion medium from normal Tyrode solution to low Na-Ca solution (Fig. 4). The I-V relation shifted still further upward and left during perfusion with low $\mathrm{Na}-\mathrm{Ca}$ solution containing verapamil. The inward going rectification shown by the I-V curve virtually disappeared during this procedure, suggesting an increase of background outward current. This shift in the I-V curve produced by verapamil was partly reversed after reperfusion with solution without the drug.

In Fig. 5, we compare the effects of d-and l-isomers of verapamil on the time course of the change in the frequency of action potential discharges. In the first $10 \mathrm{~min}$ after drug application, the percent decrease in frequency was virtually the same for both the $\mathrm{d}$ - and $\mathrm{l}$-isomer. However, after about 20 min, the l-isomer produced a significantly $(\mathrm{p}<0.05)$ greater decrease in frequency. In addition, the action potential discharge ceased completely at 25 min after application of the 1-isomer. On the other hand, at least $40 \mathrm{~min}$ were required to produce complete quiescence of the preparation when $d$ isomer was used.

It may be interesting to know which isomer the verapamil-induced upward shift in I-V curve (Fig. 4) is ascribable to, because the shift of I-V curve in this direction (e.g., due to increased $\mathrm{K}^{+}$conductance) may contribute, at least in part, to a decrease in the frequency of repetitive firing in depolarized ventricular myocardium. ${ }^{1,7)}$ Thus, we studied the effects of d-and l-isomers on the $\mathrm{I}-\mathrm{V}$ relationship in experimental situations similar to those in Fig. 3. As illustrated in Fig. 6, 1-isomer shifted the I-V relation upward and to the left (panel A) while d-isomer produced either no shift or a slight shift down-

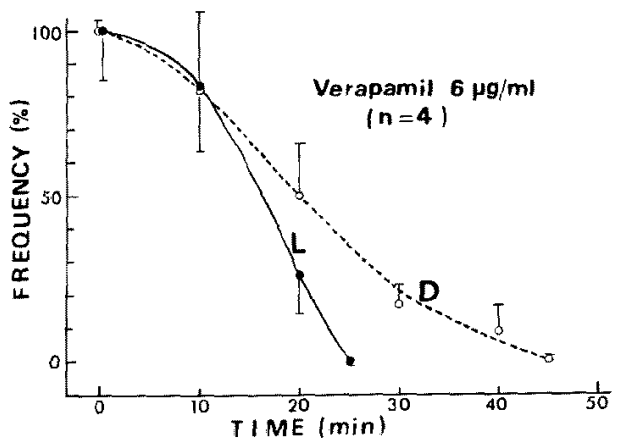

Fig. 5. Effect of 1- and d-isomers $(6 \mu \mathrm{g} / \mathrm{ml})$ of verapamil on frequency of repetitive action potential discharge. Abscissa: time course after application of the agents. Ordinate: percent change in the frequency. Solid circles and solid line: effect of 1 -isomer; open circles and broken line: effect of $\mathrm{d}$ isomer, Vertical bars indicate standard deviation of the mean calculated from 4 preparations tested. Each curve fitted by eye. 


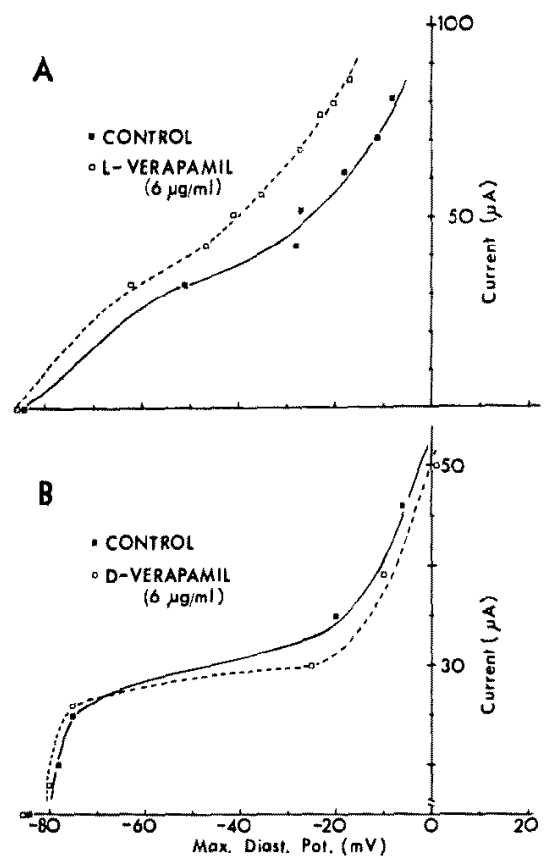

Fig. 6. Effects of 1-isomer (A) and d-isomer (B) of verapamil on currentvoltage relationship. In $A$ and $B$ : solid lines, control; dotted lines, effects of agents.

ward to the right (panel B). Similar results were obtained in the other 4 preparations tested.

\section{Discussion}

The inhibitory effects of verapamil on the electrically induced automatic discharges in Purkinje fibers, ${ }^{5)}$ and ventricular ${ }^{1,9)}$ and atrial muscle fibers ${ }^{14)}$ have been reported, but there has been no report of the effects of its optical isomers on these automatic activities. The machanism of the repetitive responses induced by depolarizing DC-currents in canine ventricular myocardium has been considered as follows: ${ }^{1), 2)}$ 1) The upstroke phase of the response depends on activation of slow inward current. 2) The pacemaker potential (phase 4 depolarization) is induced by deactivation of the time and voltage dependent outward current $\left(i_{x_{1}}\right)^{12)}$ that has been activated by a preceeding action potential. 3) Decrease of the outward background current $\left(i_{k 1}\right)$ due to anomalous rectification also contributes to the development of activity in the voltage range between -60 and $0 \mathrm{mV}$.

In the present study verapamil and its optical isomers inhibited repetitive responses (Figs. 1 and 5) although there are some difference in their efficacy. 
Among these, l-isomer was more potent than d-isomer in suppressing the frequency of discharge (Fig. 5), and the l-isomer shifted the I-V relationship upwards to the left while the d-isomer did not have such effect (Fig. 6).

In this regard, Raschack ${ }^{13 !}$ reported that $\mathrm{d}$-isomer was less potent than 1-isomer in suppressing contractile tension of cardiac muscle, indicating that l-isomer was more potent than d-isomer in blocking slow inward currents. The verapamil-induced decrease in the overshoot potential and $\dot{V}_{\max }$ of repetitive slow responses (Figs. 1 and 2) may thus be partly due to decrease of the slow inward currents. This could be one of several possible explanations why the d-isomer was less potent than the l-isomer in suppressing repetitive responses in the present study.

However, different modes of action of the d-and l-isomers on the steady state $\mathrm{I}-\mathrm{V}$ relation may also be responsible for their differences in potency. When the $I-V$ relation shifts upward and to the left, as shown in Fig. 6A, there could be 2 possibilitics as far as ionic conductance change is concerned: 1) an increase of membrane conductance to $\mathrm{K}^{+}$which has negative equilibrium potentials and 2) a decrease of membrane conductance to $\mathrm{Na}^{+}$and/or $\mathrm{Ca}^{++}$ which have positive equilibrium potentials. However, the upward shift of $\mathrm{I}-\mathrm{V}$ relation which was obtained in low $\mathrm{Na}(12 \mathrm{mM})$ and low $\mathrm{Ca}(0.45 \mathrm{mM})$ solutions with verapamil suggests that the shift is due to increased background $\mathrm{K}+$ conductance $\left(g_{\mathrm{k}}\right)$, because in these extracellular ionic concentrations, the driving force for $\mathrm{Na}^{+}$and $\mathrm{Ca}^{++}$across the cell membrane may be considerably small, and therefore their contribution to the conductance change may also be small. Similar inhibition of repetitive action potential discharges during perfusion with high extracellular $\mathrm{K}^{+}$concentrations has been reported, and the mechanism of inhibition was ascribed to an increase in $\mathrm{K}^{+}$conductance. ${ }^{1,27}$

It seems likely that the l-isomer increased background outward currents and thus offset the depolarizing effects of the intracellular positive charges which had been carried by slow inward currents and/or background inward currents. Such effects may suppress the development of phase 4 depolarization and decrease the frequency of the repetitive slow responses.

The potentially different actions of the 1 - and d-isomers on both slow inward currents, ${ }^{3), 41,10), 13)}$ and background outward $\mathrm{K}+$ currents (present study), may explain the different modes of action of these isomers on the repetitive slow responses in ventricular muscle and on the experimental ventricular arrhythmias.

\section{ACKNOWLEDGMENT}

Verapamil and its optical isomers used in this study were generous gift from Eisai Pharmaceutical Company. 


\section{REFERENCES}

1. Arita M, Nagamoto Y, Saikawa T: Automaticity and time dependent conduction disturbance in canine ventricular myocardium. Jap Circulat J 40: 1409, 1976

2. Arita M, Nagamoto $Y$, Saikawa $T$ : Spontaneous electrical activity induced by depolarizing currents in canine ventricular myocardium. Jap Heart J 17: 246, 1976

3. Bayer R, Kaufmann R, Mannhold R: Inotropic and electrophysiological actions of varapamil and D-600 in mammalian myocardium. II. Pattern of inotropic effects of optical isomers. Naunyn-Schmiedeberg's Arch Pharmacol 290: 69, 1975

4. Bayer R, Kalusche D, Kaufmann R, Mannhold R: Inotropic and electrophysiological actions of verapamil and D-600 in mammalian myocardium. III. Effects of optical isomers on transmembrane action potentails. Naunyn-Schmiedeberg's Arch Pharmacol 290: 81, 1975

5. Granefield PF, Aronson RS, Wit AL: Effect of verapamil on the normal action potential and on a calcium dependent slow response of canine cardiac Purkinje fibers. Circulat Res 34: 204, 1974

6. Hall AE, Hutter OF, Noble D: Current-voltage relations of Purkinje fibres in sodiumdeficient solutions. J Physiol 166: 255, 1963

7. Imanishi S, Surawicz B: Automatic activity in depolarized guinea pig ventricular myocardium. Characteristics and mechanism. Circulat Res 39: 751, 1976

8. Kaas RS, Tsien RW: Multiple effects of calcium antagonists on plateau currents in cardiac Purkinje fibers. J Gen Physiol 66: 169, 1975

9. Katzung B: Electrically induced automaticity in ventricular myocardium. Life Science 14: 1133,1974

10. Kaumann AJ, Serur JR: Optical isomers of verapamil on canine heart. Prevention of ventricular fibrillation induced by coronary artery occlusion, impaired atrioventricular conductance and negative inotropic effects. Naunyn-Schmiedeberg's Arch Pharmacol 294: 285, 1976

11. Kohlhardt M, Bauer B, Krause H, Fleckenstein A: Differentiation of the transmembrane $\mathrm{Na}$ and $\mathrm{Ca}$ channels in mammalian cardiac fibers by the use of specific inhibitors. Pflüg Arch 335: 309,1972

12. Noble D: The Initiation of the Heartbeat, Oxford University Press, London, 1975

13. Raschack $M$ : Relationship of antiarrhythmic to inotropic activity and antiarrhythmic qualities of the optical isomers of verapamil. Naunyn-Schmiedeberg's Arch Pharmacol 294: 285, 1976

14. Saikawa $T$, Arita M, Nagamoto $Y$ : Electrically induced automaticity in canine ventricular myocardium. Recent Advances in Studies on Cardiac Structure and Metabolism, Vol 11, Heart Function and Metabolism, ed by Kobayashi T, Sano T, Dhalla NS, p45-49, 1978 